\title{
Robust Subspace Tracking Algorithms in Signal Processing: A Brief Survey
}

\author{
Le Trung Thanh ${ }^{1,2}$, Nguyen Viet Dung ${ }^{1,3}$, Nguyen Linh Trung ${ }^{1}$, Karim Abed-Meraim ${ }^{2}$ \\ 1 AVITECH, VNU University of Engineering and Technology, Hanoi, Vietnam \\ 2 PRISME Laboratory, University of Orléans, Orléans, France \\ ${ }^{3}$ National Institute of Advanced Technologies of Brittany, Brest, France
}

Correspondence: Nguyen Linh Trung, linhtrung@vnu.edu.vn

Communication: submitted 26 May 2021, revised 22 June 2021, accepted 22 June 2021

Online publication: 23 June 2021, Digital Object Identifier: 10.21553/rev-jec.270

The associate editor coordinating the review of this article and recommending it for publication was Prof. Vo Nguyen Quoc Bao.

\begin{abstract}
Principal component analysis (PCA) and subspace estimation (SE) are popular data analysis tools and used in a wide range of applications. The main interest in PCA/SE is for dimensionality reduction and low-rank approximation purposes. The emergence of big data streams have led to several essential issues for performing PCA/SE. Among them are (i) the size of such data streams increases over time, (ii) the underlying models may be time-dependent, and (iii) problem of dealing with the uncertainty and incompleteness in data. A robust variant of PCA/SE for such data streams, namely robust online PCA or robust subspace tracking (RST), has been introduced as a good alternative. The main goal of this paper is to provide a brief survey on recent RST algorithms in signal processing. Particularly, we begin this survey by introducing the basic ideas of the RST problem. Then, different aspects of RST are reviewed with respect to different kinds of non-Gaussian noises and sparse constraints. Our own contributions on this topic are also highlighted.
\end{abstract}

Keywords- Streaming PCA, subspace tracking, robust algorithm, imperfect data, sparse outlier, impulse noise, colored noise, sparse subspace.

\section{INTRODUCTION}

Principal component analysis (PCA) and subspace estimation (SE) are widely used as a fundamental step for dimensionality reduction and analysis. Their main purpose is to extract low-dimensional subspaces from high-dimensional data while still keeping as much relevant information as possible. Consequently, PCA and SE have found success in a wide range of fields, from finance to neuroscience, with the most successful applications in computer science. The main difference between them is that PCA emphasizes the use of eigenvectors rather than of subspace as in SE. PCA in a standard set-up can be implemented by using either eigenvalue decomposition (EVD) or singular value decomposition (SVD) and is proved to be optimal in terms of the Frobenius-norm approximation error by the Eckart-Young theorem [1].

Recent years have witnessed an increasing interest in adaptive processing [2]. It is mainly due to the fact that online applications generate a huge amount of data streams over time and such streams are often with high veracity and velocity. It is known that veracity requires robust algorithms for handling imperfect data while velocity demands (near) real-time processing. Accordingly, important classes of PCA, such as subspace tracking (ST) also called PCA for streaming data or streaming PCA or dynamic PCA, and ST with missing data have drawn much research attention recently in signal processing and modern data analysis.
The attractive point of ST resides on two aspects. First, in a similar manner to batch subspace methods [3], both the main components and the disturbance components of data observation can be exploited in many different ways. In fact, the subspace is simple to understand (i.e., in a statistical sense) and implement, thus proving its efficiency in many practical applications. Second, different from batch subspace methods, ST has a better trade-off between the accuracy and the computational complexity, thus making it suitable for time-sensitivity and real-time applications. Due to its practical use, we can find a wide range of applications in diverse fields [3-5], for example, direction of arrival (DoA) tracking in radar and sonar, data compression and filtering, blind channel estimation and equalization, and pattern recognition, to name a few.

However, it is well-known that PCA/SE is very sensitive to data corruptions. This fact remains across the above important PCA classes in general and ST in particular. PCA dealing with impulsive noise and outliers is referred to as robust PCA. In 2011, it was revisited in a seminal work of Candes et al [6]. This work has attracted many research studies and applications, with over 4000 citations as of now. PCA for streaming data with impulsive noise and outliers is referred to as robust subspace tracking (RST). It is considered much more difficult than the original ST [7].

ST algorithms have been developed for over three decades [3, 4]. It has been around ten years since Delma's survey [3] and we thus believe it is not only 
Table I

Surveys on PCA/SE AND SubsPace Tracking

\begin{tabular}{|c|c|c|}
\hline Paper & Topic \& scope & Main contribution \\
\hline$[4,1990]$ & Principal ST & $\begin{array}{l}\text { A survey on numerical methods for tracking the low-rank approximation of } \\
\text { covariance matrices slowly varying with time. }\end{array}$ \\
\hline$[3,2010]$ & Principal and minor ST & A comprehensive survey on classical ST algorithms. \\
\hline$[8,2016]$ & Principal component analysis & A survey on adaptations of PCA for modern datasets and applications. \\
\hline$[9,2018]$ & Principal ST & $\begin{array}{l}\text { A high-dimensional analysis framework for the state-of-the-art ST algorithms } \\
\text { from incomplete observations. }\end{array}$ \\
\hline$[5,2018]$ & ST and streaming PCA & $\begin{array}{l}\text { A survey on both classical and recent ST algorithms able to handle missing data } \\
\text { and their performance guarantee. }\end{array}$ \\
\hline$[10,2018]$ & Robust subspace learning & $\begin{array}{l}\text { A survey on robust PCA, RST, and robust subspace recovery in the presence } \\
\text { of sparse outliers. }\end{array}$ \\
\hline$[11,2018]$ & Robust PCA & A survey on statistic and dynamic robust PCA algorithms. \\
\hline$[12,2018]$ & Principal component analysis & A survey on distributed PCA algorithms. \\
\hline$[13,2018]$ & Robust subspace recovery & $\begin{array}{l}\text { A survey on works on robust subspace recovery when measurements are } \\
\text { corrupted by sparse outliers. }\end{array}$ \\
\hline$[14,2018]$ & Sparse PCA & A survey on recent theoretical developments of sparse PCA. \\
\hline Ours & RST & $\begin{array}{l}\text { A survey on RST algorithms in the presence of different kinds of corruptions } \\
\text { (e.g. outliers, missing data, impulsive, and colored noise) and sparse subspace. }\end{array}$ \\
\hline
\end{tabular}

important but the right time to do an up-to-date survey in order to highlight some aspects that were not mentioned in [3] as well as recent advances on this topic.

\subsection{Related Work}

Due to the importance of ST, there have been a number of published surveys in the literature. One of the first and earliest surveys on principal subspace tracking algorithms was carried on by Comon and Golub in [4]. The survey focuses on methods with high and moderate computational complexity for tracking the lowrank approximation of covariance matrices which may be slowly varying with time. In [3], Delmas provided a comprehensive overview on developments of classical ST algorithms with low (linear) complexity.

Recently, different adaptations of PCA for modern datasets and applications were reviewed in [8]. However, PCA for streaming data or ST was not addressed. The problem of tracking the underlying subspace of data from incomplete observations was discussed in [5] and [9]. Particularly, the former concerned methodological classes of ST algorithms that are able to deal with missing data while the latter presented a highdimensional framework for analyzing their convergence behavior. The survey in [10] carried out reviews on robust PCA, RST, and robust subspace recovery in the presence of sparse outliers. Two similar surveys to [10] have also been conducted in [11] and [13] which respectively review (i) static and dynamic RPCA algorithms, and (ii) the entire body of works on robust sparse recovery. In the literature, there exist two others surveys on two adaptations of PCA which are distributed PCA [12] and sparse PCA [14].
The main contributions of the above-mentioned papers are summarized in Table I.

\subsection{Main Contributions}

To the best of our knowledge, we are not aware of any work that reviews the RST problem in the presence of different kinds of non-Gaussian noise. Although the three surveys $[10,11,13]$ reviewed some classes of RST algorithms, they only discussed on sparse outliers. Methods for other non-Gaussian noises (e.g., impulsive noise and colored noise) have not been reviewed yet. Moreover, no survey exists on the problem of sparse $\mathrm{ST}$ in the literature. This observation motivates us to carry out a survey on the topic.

The main goal of this survey is to fill the gap in the literature addressing the following three kinds of nonGaussian noises (including outliers, impulsive noise, and colored noise) and sparse constraints. Our contributions are as follows. First, in the context of missing data and outliers, we review four main approaches for dealing with them. They are Grassmannian, recursive least-squares (RLS), recursive projected compressive sensing (ReProCS), and adaptive projected subgradient method (APSM). Second, when the measurements are corrupted by impulsive noise, we show that most of state-of-the-art RST algorithms are based on improving the well-known PAST algorithm which belongs to the class of RLS methods. Two other appealing approaches including weighted RLS and adaptive Kalman filtering are also reviewed. Third, we outline two main classes of RST algorithms that are able to deal with colored noise: instrumental variable-based and oblique projections. Finally, a short review on sparse ST algorithms is presented. 
The structure of our review is as follows. Section 2 states the problem of RST. In Section 3, we provide the state-of-the-art algorithms for the RST problem in the presence of missing data and outliers. The next two sections, 4 and 5, present RST algorithms that are able to handle impulsive noise and colored noise, respectively. Section 6 provides a short review on sparse ST. Finally, Section 7 concludes the paper.

\section{Robust Subspace Tracking: Problem FORMULATION}

At each time $t$, we suppose to observe a signal $\mathbf{x}_{t} \in \mathbb{R}^{n}$ satisfying

$$
\mathbf{x}_{t}=\mathbf{P}_{t} \ell_{t}+\mathbf{P}_{t} \mathbf{v}_{t}
$$

where $\mathbf{P}_{t} \in \mathbb{R}^{n \times n}$ is an observation mask matrix indicating the $i$-th entry of $\mathbf{x}_{t}$ is observed (i.e., $\mathbf{P}_{t}(i, i)=1$ ) or not (i.e., $\left.\mathbf{P}_{t}(i, i)=0\right), \mathbf{v}_{t} \in \mathbb{R}^{n \times 1}$ is the (non-Gaussian) noise vector and $\ell_{t}$ is the true signal living in a fixed or slowly time-varying low-dimensional subspace of $\mathbb{R}^{n}$. More concretely, $\ell_{t}=\mathbf{U}_{t} \mathbf{w}_{t}$ in which $\mathbf{w}_{t}$ is a weight vector and $\mathbf{U}_{t} \in \mathbb{R}^{n \times r}(r \ll n)$ is a basis matrix with $d\left(\mathbf{U}_{t}, \mathbf{U}_{t-1}\right) \triangleq \sin \left(\theta\left(\mathbf{U}_{t}, \mathbf{U}_{t-1}\right)\right) \ll 1$ where $\theta\left(\mathbf{U}_{t}, \mathbf{U}_{t-1}\right)$ denotes the largest principal angle between $\mathbf{U}_{t}$ and $\mathbf{U}_{t-1}$.

The RST problem can be stated as follows: Given a streaming set of observed signals $\left\{\mathbf{x}_{t}\right\}_{t>1}$ in (1), we wish to estimate a rank-r matrix $\mathbf{U}_{t}$ such that it can cover the span of the complete-data noiseless signal $\boldsymbol{\ell}_{t}$.

In this paper, we consider the RST problem in the presence of different kinds of the non-Gaussian noise $\mathbf{v}_{t}$ : sparse outliers, impulse noise, and colored noise. Also, we review sparse ST algorithms under the constraint that the basis matrix $\mathbf{U}_{t}$ is sparse.

\section{Robust Subspace Tracking IN THE Presence of Missing Data \& Outliers}

In the literature, there have been several studies on ST in the presence of outliers and missing data. The proposed RST algorithms can be categorized into four main classes: (i) Grassmannian, (ii) recursive eastSquares (RLS), (iii) recursive projected compressive sensing (ReProCS), and (iv) adaptive projected subgradient method (APSM). We summarize all the RST algorithms robust to outliers and missing data in Table II.

\subsection{Grassmannian Algorithms}

Many of RST algorithms are based on the Grassmannian approach in which the ST procedure can be cast into an optimization process on a Grassmann manifold. More concretely, Grassman manifold is a space that parameterizes all $r$-dimensional linear subspaces of the $N$-dimensional vector space. The underlying subspace can be derived from averaging the column span of the (fully or partially) observed signals on the Grassmannian. Interestingly, each observed signal $\ell_{t}$ spans a one-dimensional subspace which can be described as a point in the Grassmannian. Therefore, the Grassmannian approach offers several advantages such as a lower number of parameters to optimize and limited memory usage and the resulting RST algorithms are often efficient and scalable to high dimensional data [37].

State-of-the-art RST algorithms include GRASTA [15], GOSUS [16], pROST [17, 18], and RoIGA [33, 34]. In [15], He et al. proposed an efficient RST algorithm called Grassmannian robust adaptive ST (GRASTA) which is a robust version of GROUSE in [38]. GRASTA first uses an $\ell_{1}$-norm cost function to reduce the effect of sparse outliers and then performs the incremental gradient on the Grassmann manifold of the subspace U. In [16], Xu et al. introduced an effective algorithm namely GOSUS for tracking subspace with structuredsparsity. GOSUS also incorporates an adaptive stepsize for the incremental gradient on the manifold. The effectiveness of GOSUS was demonstrated via the real application of video background subtraction and multiple face tracking. In [17, 18], Hage et al. proposed a method, namely pPOST that combines the advantages of Grassmannian optimization with a non-convex sparsity measure. Instead of using the $\ell_{1}$ norm regularization, pPOST uses the penalty with nonconvex $\ell_{0}$-surrogates allows reconstruction even in the case when $\ell_{1}$-based methods fail. Another algorithm dubbed robust intrinsic Grassmann average (RoIGA) was proposed by Rudrasis et al. in [33, 34]. RoIGA is a geometric approach to computing principal linear subspaces in finite and infinite dimensional reproducing kernel Hilbert spaces. Among them, RoIGA is shown as one of the fastest RST algorithms for handling missing data corrupted by outliers.

\subsection{Recursive Least-Squares based Algorithms}

Another line of the RST research is based on recursive least-squares (RLS) methods where the underlying subspace is recursively updated by minimizing a (weighted) least-squares objective function containing squared residuals and a penalty accounting for outliers. An efficient RLS-based algorithm is parallel estimation and tracking by recursive least squares (PETRELS) [39] which can be considered as an extension of the projection approximation ST (PAST) algorithm [40] in order to handle missing data.

Inspired by PETRELS, several robust variants have been proposed to deal with outliers the same line such as [20, 27, 35, 36]. Robust online subspace estimation and tracking (ROSETA) in [20] applies an adaptive step size at the stage of subspace estimation to enhance the convergence rate. Meanwhile the main idea of PETRELS-CFAR algorithm [27] is to handle "outliers-removed" data (i.e., outliers are first removed before performing ST) using a Constant False Alarm Rate (CFAR) detector. Adopting the approach of PETRELS-CFAR, but aiming to improve RST performance, we proposed an efficient algorithm called PETRELS-ADMM which is able to remove outliers more effectively in $[35,36]$. It includes two main stages: outlier rejection and subspace estimation and tracking. 
Table II

RST Algorithms in the Presence of Both Missing Data and Sparse Outliers

\begin{tabular}{|c|c|c|c|c|c|c|c|}
\hline Algorithm & Method & $\begin{array}{l}\text { Missing } \\
\text { Data }\end{array}$ & Outliers & $\begin{array}{c}\text { Prior } \\
\text { Information }\end{array}$ & Initialization & $\begin{array}{l}\text { Convergence } \\
\text { Guarantee }\end{array}$ & $\begin{array}{l}\text { Computational } \\
\text { Complexity }\end{array}$ \\
\hline $\begin{array}{l}\text { GRASTA } \\
(2012[15])\end{array}$ & $\begin{array}{l}\ell_{1} \text {-norm }+ \text { ADMM + } \\
\text { Grassmannian }\end{array}$ & $\checkmark$ & $\checkmark$ & $x$ & random & $x$ & $\mathcal{O}\left(n r+r^{3}\right)$ \\
\hline $\begin{array}{l}\text { GOSUS } \\
(2014[16])\end{array}$ & $\begin{array}{l}\ell_{2} \text {-norm + ADMM + } \\
\text { Grassmannian }\end{array}$ & $x$ & $\checkmark$ & $\checkmark$ & random & $x$ & - \\
\hline $\begin{array}{l}\text { pROST } \\
(2014[17,18])\end{array}$ & $\begin{array}{l}\ell_{0} \text {-surrogate }+ \text { Grassmannian } \\
\text { Conjugate Gradient }\end{array}$ & $x$ & $\checkmark$ & $x$ & random & $x$ & - \\
\hline $\begin{array}{l}\text { MRMD } \\
\text { (2014 [19]) }\end{array}$ & $\begin{array}{l}\text { Online max-norm } \\
\text { regularization }\end{array}$ & $x$ & $\checkmark$ & $\checkmark$ & random & $\checkmark$ & - \\
\hline $\begin{array}{l}\text { ROSETA } \\
(2015[20])\end{array}$ & $\begin{array}{l}\ell_{1,2} \text {-norm + ADMM + } \\
\text { RLS }\end{array}$ & $\checkmark$ & $\checkmark$ & $x$ & random & $x$ & $\mathcal{O}\left(n r^{2}\right)$ \\
\hline $\begin{array}{l}\text { Roubst STAPSM } \\
(2015[21,22])\end{array}$ & APSM + CoSAMP* & $\checkmark$ & $\checkmark$ & $x$ & random & $\checkmark$ & $\mathcal{O}\left(k n r^{2}\right)$ \\
\hline $\begin{array}{l}\text { ReProCS-cPCA } \\
(2016 \text { [23]) }\end{array}$ & ReProCS & $x$ & $\checkmark$ & $\checkmark$ & batch & $\checkmark$ & $\mathcal{O}\left(n r \log ^{2}(n) \log (1 / \epsilon)\right)^{\diamond}$ \\
\hline $\begin{array}{l}\text { OTNNR } \\
(2016[24])\end{array}$ & $\begin{array}{l}\text { Truncated nuclear-norm } \\
\text { regularization }\end{array}$ & $x$ & $\checkmark$ & $x$ & random & $x$ & - \\
\hline $\begin{array}{l}\text { OLP-RPCA } \\
(2017 \text { [25]) }\end{array}$ & $\begin{array}{l}\ell_{p} \text {-norm }+ \text { singular } \\
\text { value thresholding }\end{array}$ & $x$ & $\checkmark$ & $x$ & random & $\checkmark$ & $\mathcal{O}\left(n r+r^{3}\right)$ \\
\hline $\begin{array}{l}\text { L1-PCA } \\
(2018[26])\end{array}$ & $\begin{array}{l}\ell_{1} \text {-norm }+ \\
\text { Bit-flipping }\end{array}$ & $x$ & $\checkmark$ & $x$ & batch & $x$ & $\mathcal{O}\left(n r \omega^{2}\right)^{\ddagger}$ \\
\hline $\begin{array}{l}\text { PETRELS-CFAR } \\
(2018 \text { [27]) }\end{array}$ & Robust statistic + RLS & $\checkmark$ & $\checkmark$ & $\checkmark$ & batch & $x$ & $\mathcal{O}\left(n r^{2}+n \omega\right)^{+\dagger}$ \\
\hline $\begin{array}{l}\text { s-ReProCS } \\
(2019[28])\end{array}$ & ReProCS & $\checkmark$ & $\checkmark$ & $\checkmark$ & batch & $\checkmark$ & $\mathcal{O}(n r \log (n) \log (1 / \epsilon))^{\diamond}$ \\
\hline $\begin{array}{l}\text { NORST-miss } \\
(2019 \text { [29]) }\end{array}$ & ReProCS & $\checkmark$ & $x$ & $\checkmark$ & batch & $\checkmark$ & $\mathcal{O}(n r \log (1 / \epsilon))^{\diamond}$ \\
\hline $\begin{array}{l}\text { L1-IRW } \\
(2019 \text { [30]) }\end{array}$ & $\begin{array}{l}\ell_{1} \text {-norm }+ \\
\text { Bit-flipping }\end{array}$ & $x$ & $\checkmark$ & $x$ & batch & $x$ & $\mathcal{O}\left(k\left(n \omega r^{3} p+2^{r} n r^{2}\right)\right)^{\dagger}$ \\
\hline $\begin{array}{l}\text { OSTP } \\
(2019 \text { [31]) }\end{array}$ & $\begin{array}{l}\text { Schatten quasi-norm }+ \\
\text { Block-proximal gradient }\end{array}$ & $x$ & $\checkmark$ & $x$ & random & $\checkmark$ & $\mathcal{O}\left(n r^{2}\right)$ \\
\hline $\begin{array}{l}\text { NORST } \\
(2020[32])\end{array}$ & ReProCS & $\checkmark$ & $\checkmark$ & $\checkmark$ & batch & $\checkmark$ & $\mathcal{O}(n r \log (1 / \epsilon))^{\diamond}$ \\
\hline $\begin{array}{l}\text { RoIGA } \\
(2020[33,34])\end{array}$ & $\mathrm{IGA}^{\#}+$ Grassmannian & $x$ & $\checkmark$ & $x$ & random & $x$ & - \\
\hline $\begin{array}{l}\text { PETRELS-ADMM } \\
(2021[35,36])\end{array}$ & $\begin{array}{l}\ell_{1} \text {-norm + ADMM + } \\
\text { RLS }\end{array}$ & $\checkmark$ & $\checkmark$ & $\checkmark$ & random & $\checkmark$ & $\mathcal{O}\left(n r^{2}\right)$ \\
\hline $\begin{array}{l}{ }^{*} \text { CoSAMP: Compr } \\
{ }^{\circ} \epsilon: \text { a desired subs } \\
++\omega \text { : length of trai }\end{array}$ & $\begin{array}{l}\text { essed Sampling Orthogonal M } \\
\text { oace recovery accuracy } \\
\text { ing window }\end{array}$ & Pur & $\begin{array}{l}\text { " IGA: } \\
\ddagger \omega: \text { ler } \\
+\omega \text { : ler }\end{array}$ & $\begin{array}{l}\text { trinsic Grassn } \\
\text { th of sliding } \\
\text { th of sliding }\end{array}$ & $\begin{array}{l}\text { ann Average } \\
\text { indow } \\
\text { indow; } k \text { : numl }\end{array}$ & r of iterations; & : number of bit flips \\
\hline
\end{tabular}

Outliers living in the measurement data are detected and removed by a ADMM solver in an effective way. An improved PETRELS was then introduced to update the underlying subspace. In practice, the convergence rate of RST-type algorithms is often faster than that of Grassmmannian-based algorithms in slowly timevarying environments.

\subsection{Recursive Projected Compressive Sensing based Algorithms}

Recursive projected compressive sensing (ReProCS)based algorithms [23, 28, 29, 32] are also capable of tracking subspace in the presence of outliers and missing data.
ReProCS-type algorithms use the piecewise constant subspace change model described previously and start with a "good" estimate of the initial subspace. At each time, they first solve a projected compressive sensing problem to derive the sparse outliers, e.g., using $\ell_{1}$ minimization followed by thresholding-based support estimation. After that, the subspace direction change is then estimated by using projection-SVD [28].

ReProCS provides not only a memory-efficient and highly robust solution, but also a precise subspace estimation compared to the state-of-the-arts. However, ReProCS-type algorithms often require strong assumptions on subspace changes, outlier magnitudes, and accurate initialization. 
Table III

RST Algorithms in the Presence of Impulsive Noise

\begin{tabular}{|c|c|c|c|c|c|c|c|}
\hline Algorithm & Method & $\begin{array}{l}\text { Burst } \\
\text { noise }\end{array}$ & $\begin{array}{l}\text { SIRV } \\
\text { noise }\end{array}$ & $\begin{array}{c}\alpha \text {-stable } \\
\text { noise }\end{array}$ & Initialization & $\begin{array}{c}\text { Convergence } \\
\text { Guarantee }\end{array}$ & $\begin{array}{l}\text { Computational } \\
\text { Complexity }\end{array}$ \\
\hline $\begin{array}{l}\text { RPAST } \\
(2006[41])\end{array}$ & PAST + M-estimation & $\checkmark$ & - & $\checkmark$ & random & $\checkmark$ & $\mathcal{O}\left(n r+r^{2}\right)$ \\
\hline $\begin{array}{l}\text { MCC-PAST } \\
(2014[42])\end{array}$ & $\begin{array}{l}\text { Maximum correntropy } \\
\text { criterion (MCC) + PAST }\end{array}$ & $\checkmark$ & - & $\checkmark$ & random & $x$ & $\mathcal{O}\left(n r+r^{2}\right)$ \\
\hline $\begin{array}{l}\text { BNC-PAST } \\
(2014[43])\end{array}$ & $\begin{array}{l}\text { Bounded nonlinear } \\
\text { covariance (BNC) + PAST }\end{array}$ & $\checkmark$ & - & $\checkmark$ & random & $x$ & $\mathcal{O}\left(n r+r^{2}\right)$ \\
\hline $\begin{array}{l}\text { robust KFVM } \\
(2020[44])\end{array}$ & $\begin{array}{l}\text { Adaptive Kalman filter + } \\
\text { M-estimation }\end{array}$ & $\checkmark$ & - & - & random & $x$ & $\begin{array}{l}\mathcal{O}\left(n r \ell+\ell r^{2}\right)+ \\
\mathcal{O}\left(\ell^{2} r+\ell^{3}\right)\end{array}$ \\
\hline $\begin{array}{l}\text { ROBUSTA } \\
(2018 \text { [27]) }\end{array}$ & $\begin{array}{l}\text { Weighted RLS }+ \\
\text { Mahalanobis distance }\end{array}$ & $\checkmark$ & $\checkmark$ & $\checkmark$ & random & $\checkmark$ & $\mathcal{O}\left(n r+r^{2}\right)$ \\
\hline
\end{tabular}

$\ell$ : length of the sliding window

-: unknown or undetermined

\subsection{Adaptive Projected Subgradient Method based Algorithms}

Adaptive projected subgradient method (APSM) can provide a robust solution to the presence of missing data and outliers [21, 22]. Main advantages of APSM are that convex constraints can be readily incorporated and it can be used as an alternative to constructing the cost function from the sum of square errors like RLS methods. The key idea of APSM stems from that unknown parameters of regression models can be estimated from seeking a point in the intersection of all the sets defined by measurements. In the context of ST, based on the latest observed signals, a cost function is properly chosen at each time instant which scores a zero loss. The next task is to reach the intersection point. To deal with sparse outliers, APSM-type algorithms detect the time instances at which the observed signals are corrupted by outliers via using sparsityaware greedy techniques (e.g. compressed sampling orthogonal matching pursuit as used in [22]) and then reject them.

\subsection{Other Algorithms}

Some other RST algorithms are able to track the underlying subspace over time from measurements corrupted by sparse outliers such as MRMD [19], OTNNR [24], L1-PCA [26], L1-IRW [30], OLPRPCA [25], and OSTP [31]. Most of them use a $\ell_{p^{-}}$ regularization $(0 \leq p \leq 1)$ to discard the effect of outliers. However, they are not designed for missing data.

\section{Robust Subspace Tracking IN the Presence of IMpulsive Noise}

By "impulsive", we mean it can be burst noise $[45,46]$, spherically invariant random variable (SIRV) noise [47, $48]$, or alpha-stable noise [49,50]. We note that even though these algorithms were described to reduce the effect of impulsive noise in general, most simulation results were shown for burst noise only. RST algorithms that are robust to impulsive noise are summarized in Table III.

\subsection{Robust Variants of PAST}

To take into account impulsive noise, some methods proposed in the literature have mainly been based on robust statistics so far. Among them, some studies have proposed robust variants of PAST to deal with impulsive noise. In [41], a robust PAST (RPAST) was proposed. The algorithm first detects the occurrence of the impulsive noise based on a threshold, and then eliminates undesirable effects by discarding contaminated observations. The threshold is determined based on an empirical function of noise variance with the assumption that error vectors follow a Gaussian distribution corrupted by additive impulsive noise.

Zhang et al. introduced another PAST's variant called MCC-PAST via the maximum correntropy criterion (MCC) in [42, 51, 52]. MCC-PAST exploits a correntropy as a new statistic, which can quantify both the time structures and statistics of two random processes, to deal with impulsive noise. Accordingly, the maximum correntropy criterion (MCC) is applied as a substitute for the mean square error criterion in the objective function of PAST. Based on the RLS technique, the MCC-PAST algorithm was then developed. To extend the tracking capability of the MCC-PAS, a variable forgetting factor (FF) technique was also employed in the recursion process. In parallel, Shengyang et al. developed another robust variant of PAST, namely BNCPAST, to track the underlying subspace via a different criterion [43]. The authors defined a new concept namely bounded non-linear covariance (BNC) to handle relative problems (including ST) in the presence of non-Gaussian noise with a heavy-tailed distribution. In particular, bounded nonlinear maps were employed to discard the effect of impulsive noise. Accordingly, a new robust PAST algorithm based on BNC was derived.

\subsection{Adaptive Kalman Filtering}

Another good approach capable of handling impulsive noise is based on adaptive Kalaman filtering. In [44], Liao et al. proposed a RST algorithm based on an adaptive Kalman filter with variable number of measurements (KFVM). The main idea of using the 
Table IV

RST Algorithms in the Presence of Colored Noise

\begin{tabular}{llccc}
\hline Method & Approach & Initialization & $\begin{array}{c}\text { Convergence } \\
\text { Guarantee }\end{array}$ & $\begin{array}{l}\text { Computational } \\
\text { Complexity }\end{array}$ \\
\hline $\begin{array}{l}\text { IV-PAST } \\
(2012[53])\end{array}$ & IV + PAST & random & $\boldsymbol{x}$ & $3 n \ell+\mathcal{O}(n r)$ \\
\hline $\begin{array}{l}\text { IVPM } \\
(2014 \text { [54] })\end{array}$ & IV + propagator-based & random & $\boldsymbol{x}$ & $n(\ell+2 r)$ \\
\hline $\begin{array}{l}\text { LOFF-VR-SREIV-PAST } \\
(2020 \text { [55] })\end{array}$ & $\begin{array}{l}\text { IV + PAST + } \\
\text { adaptive forgetting factor }\end{array}$ & random & $\checkmark$ & $\begin{array}{l}6 n r+5 r^{2}+4 n \\
+14 r+\mathcal{O}(n r)\end{array}$ \\
\hline $\begin{array}{l}\text { obPAST } \\
(2005 \text { [56] })\end{array}$ & $\begin{array}{l}\text { Oblique projection }+ \\
\text { PAST }\end{array}$ & random & $\boldsymbol{x}$ & $3 n r^{2}+3 n r+\mathcal{O}\left(r^{3}\right)$ \\
\hline $\begin{array}{l}\text { obYAST } \\
(2012[57])\end{array}$ & $\begin{array}{l}\text { Oblique projection }+ \\
\text { YAST }\end{array}$ & random & $\boldsymbol{x}$ & $5 n r+\mathcal{O}\left(r^{2}+n\right)+\mathcal{O}\left(r^{3}\right)$ \\
\hline
\end{tabular}

$\ell$ : the dimension of IV vector.

KFVM is to deal with the tracking of fast-varying subspace [58]. More concretely, when the underlying subspace varies quickly, a small number of past observations are exploited in the recursion and vice versa. To handle the impulsive noise, the M-estimate technique is incorporated into the KFVNM algorithm. The complexity of the proposed KFVM-based algorithm is much higher than the PAST-based algorithms especially when the number of observations used for subspace update is large.

\subsection{Weighted Recursive Least-Squares Method}

Recently, based on robust statistics but different from the common two-step scheme mentioned above, we proposed in [27] an RST algorithm with linear computational complexity based on a weighted RLS approach, namely ROBUSTA. On the theoretical aspect, we provided a converge analysis of ROBUSTA in the presence of SIRV noise. Interestingly, we showed that it also corresponded to adaptive robust covariance estimation. ROBUSTA outperformed many state-of-the-art algorithms for burst noise, SIRV noise, and alpha-stable noise. Also, it can be easily adapted, in conjunction with pre-processing steps, to handle alpha-stable noise.

\section{Robust Subspace Tracking IN the Presence of Colored Noise}

In the literature, RST algorithms that are robust to colored noise can be categorized into two groups: (i) instrumental variable and (ii) oblique projection. We summarize these algorithms in Table IV.

\subsection{Instrumental Variable based Algorithms}

For colored noise, one of the main directions is to use the instrumental variable (IV) which allows avoiding biased estimate. An appealing benefit of this approach is easy to adapt derivation from classical ST algorithms. While having improved performance, the computational complexity of IV-based algorithms is often higher than the original ones due to the selection of the IV vector size. Specifically, in [53], two direct extensions of the PAST algorithms, named IV-PAST and extended IV-PAST, were proposed. It is shown that their performance is enhanced, comparing to the original ones. With the aim to improve further performance in subspace-based system identification applications, several algorithms in conjunction with using IV were addressed in [54]. The key idea is to adapt the propagator approach by exploiting the relationship between array signal processing and subspace identification.

Very recently, Chan et al. in [55] proposed a new robust variant of PAST capable of handing linear models with complex coefficients, multiple outputs, and colored noises. In the proposed method, the authors used a new adaptive forgetting factor and imposed a $\ell_{2}$-norm regularization into the objective function of PAST. In particular, the adaptive forgetting factor was obtained at each time instant by minimizing the meansquare deviation of the estimator from an extended IV linear model and IV-PAST. The additional $\ell_{2}$-norm regularized term on the weight vectors is aimed to reduce the error variance and prevent the ill-conditioned computation at low SNR levels. Generally, if low computational complexity is concerned, IV-based methods require a IV vector uncorrelated with the noise which is not always met in practice.

\subsection{Oblique Projection based Algorithms}

Another direction, which can avoid the above drawback, is based on oblique projection onto the subspace manifold, such as [56, 57]. It is due to the fact that the noise vector may lie in a low dimension subspace instead of being treated as full rank in the observation space. Naturally, oblique projections arise in the solution to recover the signal. Accordingly, Chen et al. proposed a variant of PAST named oblique PAST (obPAST) to track the signal subspace in [56]. In the same line, based on the well-known YAST algorithm [65], Florian et al. introduced the new obYAST algorithm in [57]. 
Table V

SPARSE ST Algorithms

\begin{tabular}{|c|c|c|c|c|c|}
\hline Algorithm & Method & $\begin{array}{c}\text { Prior } \\
\text { Information }\end{array}$ & Initialization & $\begin{array}{c}\text { Convergence } \\
\text { Guarantee }\end{array}$ & $\begin{array}{l}\text { Computational } \\
\text { Complexity }\end{array}$ \\
\hline $\begin{array}{l}\text { OIST } \\
(2016 \text { [59]) }\end{array}$ & $\begin{array}{l}\text { Oja method }+ \\
\text { soft-thresholding }\end{array}$ & $x$ & random & $\checkmark$ & $\mathcal{O}(n r)$ \\
\hline $\begin{array}{l}\text { Streaming SPCA } \\
(2015[60])\end{array}$ & $\begin{array}{l}\text { Row truncation }+ \\
\text { QR decomposition }\end{array}$ & $x$ & batch & $\checkmark$ & $\mathcal{O}(n r \min (r, s \log n))$ \\
\hline $\begin{array}{l}\ell_{1} \text {-PAST } \\
(2016[61])\end{array}$ & $\begin{array}{l}\text { PAST method }+\ell_{1} \text {-norm } \\
\text { sample matrix inverse }\end{array}$ & $\checkmark$ & random & $x$ & $3 n r^{2}+3 n r+\mathcal{O}\left(r^{2}\right)$ \\
\hline $\begin{array}{l}\text { OVBSL } \\
(2017 \text { [62]) }\end{array}$ & $\begin{array}{l}\text { Bayesian inference }+ \\
\ell_{2} / \ell_{1} \text {-norm promotion }\end{array}$ & $\checkmark$ & random & $x$ & $\mathcal{O}\left(n r^{2}+n r\right)$ \\
\hline $\begin{array}{l}\text { SS/DS-OPAST } \\
(2017 \text { [63]) }\end{array}$ & $\begin{array}{l}\text { 2-step approach }+ \text { OPAST }+ \\
\ell_{1} \text {-norm approximation }\end{array}$ & $x$ & random & $x$ & $\begin{array}{l}3 n r^{2}+3 n r+\mathcal{O}\left(r^{3}\right) / \\
3 n r+\mathcal{O}\left(n r^{2}\right)\end{array}$ \\
\hline $\begin{array}{l}\text { SS/GSS-FAPI } \\
(2020[64])\end{array}$ & $\begin{array}{l}\text { 2-step approach }+ \text { FAPI }+ \\
\text { Givens rotations }\end{array}$ & $x$ & random & $\checkmark$ & $\begin{array}{l}2 n r^{2}+4 n r+\mathcal{O}\left(r^{2}\right) / \\
4 n r+4 n s+\mathcal{O}\left(r^{2}\right)\end{array}$ \\
\hline
\end{tabular}

Both obPAST and obYAST minimized a new exponential least-squares cost function where the orthogonal projection in the residual error term is replaced with an oblique one. Experiment results indicate that this modification can facilitate the tracking ability of PAST and YAST in the presence of colored noise. Table IV reports further information about these RST algorithms, e.g. convergence and complexity.

\section{Sparse Subspace Tracking}

Recently, sparse subspace estimation and tracking have been attracted more attention from the signal processing community due to the fact that many modern datasets admit sparse representation has huge potential capabilities for analyzing them [66]. Although several algorithms have been introduced for sparse subspace estimation in the batch setting (see [67-69] for examples), there exist only a few studies on sparse ST algorithms so far.

In [59], Chuang and Yue proposed an adaptive algorithm called OIST (which stands for Oja's algorithm with Iterative Soft Thresholding) for online sparse PCA. The authors investigated a rank-one spiked model in a high-dimension regime and indicated that the estimate of the eigenvector from the sample covariance matrix is inconsistent. To alleviate it, they introduced an extended version of Oja's algorithm followed by a softthresholding step to promote sparsity on the estimate. The asymptotic convergence, steady state, and phase transition of OIST were also derived to understand its behavior in a high-dimension regime when the dimension is much larger than the number of observations. However, OIST is designed for only rank-one subspaces, i.e. lines. In parallel, a novel online sparse PCA algorithm able to deal with rank- $k$ spiked models $(k \geq 1)$ was proposed via row truncation technique in [60]. More concretely, a simple $\ell_{2}$-norm based row truncation operator was introduced to zero out rows whose leverage score is below a predefined threshold. At each time instant, the $\mathrm{QR}$ decomposition of the resulting truncated covariance matrix was realized to update the principal subspace. The authors also proved that the proposed algorithm is consistent in the highdimension regime.

In [61], Xiaopeng et al. introduced a new robust variant of PAST called $\ell_{1}$-PAST. Specifically, the authors modified the cost function of PAST by adding a $\ell_{1}$-norm constraint imposed on the subspace matrix to control its sparsity. Accordingly, a new RLS algorithm like PAST was derived to minimize the proposed objective function in an efficient way. The $\ell_{1}$-PAST is robust and stable even when the number of observations is small.

In [62], Giampouras et al. developed a novel robust sparse ST method namely OVBSL in the lens of Bayesian inference. To deal with the sparsity constraint on the subspace matrix, OVBSL utilized the groupsparsity inducing the convex $\ell_{2} / \ell_{1}$-norm. Since it belongs to the family of Bayesian methods, no fine-tuning parameter is required and the proposed algorithm is fully automated.

In this topic, we also proposed several two-stage approach based algorithms for sparse ST in [63, 64, 70]. The main steps of the two-stage approach is as follows. We first utilize a well-known ST algorithm from the literature (e.g. PAST or FAPI) to extract an orthonormal basis of the underlying subspace. Then, we estimate a sparse weight matrix based on some criteria on sparsity such that it can span the same subspace. For example, in [63], two new algorithms SS-OPAST and DS-OPAST were designed for sparse system matrix and sparse source signals respectively. We particularly exploited the natural gradient to find the sparsest matrix from the estimated orthonormal matrix by OPAST. In [64, 70], we used FAPI in the first stage and then derived SSFAPI, orthogonal SS-FAPI, and GSS-FAPI algorithms. Specifically, the sparsity criterion considered there is differentiable and smoother than the previous one in [63]. Accordingly, it facilitates the optimization by employing the Newton method and Taylor expansions. To sum up, a performance comparison among these sparse ST algorithms is given in Table V. 


\section{Conclusions}

ST has shown an increased interest in signal processing with the aim of analyzing real-time big data problems and its improvement is in parallel to recent advances in optimization. In this paper, we provided a brief survey on adaptive algorithms for RST which were mostly developed over the last decade. We highlighted three classes of RST algorithms for dealing with nonGaussian noises including sparse outliers, impulsive noise, and colored noise. The last decade has also witnessed the widespread of high-dimensional data analysis in which sparse representation-based methods are successfully applied to many signal processing applications. Accordingly, sparse ST algorithms are also reviewed in our survey.

\section{ACKNOWLEDGMENTS}

This work was supported by the National Foundation for Science and Technology Development of Vietnam under Grant No. 102.04-2019.14.

\section{REFERENCES}

[1] I. Jolliffe, Principal Component Analysis, ser. Springer Series in Statistics. Springer-Verlag New York, 2002.

[2] T. Kolajo, O. Daramola, and A. Adebiyi, "Big data stream analysis: A systematic literature review," Journal Big Data, vol. 6, no. 1, pp. 1-30, 2019.

[3] J. P. Delmas, "Subspace tracking for signal processing," Adaptive Signal Processing: Next Generation Solutions, pp. 211-270, 2010.

[4] P. Comon and G. H. Golub, "Tracking a few extreme singular values and vectors in signal processing," Proceedings of the IEEE, vol. 78, no. 8, pp. 1327-1343, 1990.

[5] L. Balzano, Y. Chi, and Y. M. Lu, "Streaming PCA and subspace tracking: The missing data case," Proceedings of the IEEE, vol. 106, no. 8, pp. 1293-1310, Aug 2018.

[6] E. J. Candès, X. Li, Y. Ma, and J. Wright, "Robust principal component analysis?" Journal of the ACM, vol. 58, no. 3, p. 11, 2011.

[7] N. Vaswani, Y. Chi, and T. Bouwmans, "Rethinking PCA for modern data sets: Theory, algorithms, and applications," Proceedings of the IEEE, vol. 106, no. 8, pp. 12741276, Aug 2018.

[8] I. T. Jolliffe and J. Cadima, "Principal component analysis: a review and recent developments," Philosophical Transactions of the Royal Society A, vol. 374, no. 2065, p. 20150202, 2016.

[9] C. Wang, Y. C. Eldar, and Y. M. Lu, "Subspace estimation from incomplete observations: A high-dimensional analysis," IEEE Journal on Selected Topics Signal Processing, vol. 12, no. 6, pp. 1240-1252, 2018.

[10] N. Vaswani, T. Bouwmans, S. Javed, and P. Narayanamurthy, "Robust subspace learning: Robust PCA, robust subspace tracking, and robust subspace recovery," IEEE Signal Processing Magazine, vol. 35, no. 4, pp. 32-55, July 2018.

[11] N. Vaswani and P. Narayanamurthy, "Static and dynamic robust PCA and matrix completion: A review," Proceedings of the IEEE, vol. 106, no. 8, pp. 1359-1379, Aug 2018.

[12] S. X. Wu, H. Wai, L. Li, and A. Scaglione, "A review of distributed algorithms for principal component analysis," Proceedings of the IEEE, vol. 106, no. 8, pp. 13211340, Aug 2018.

[13] G. Lerman and T. Maunu, "An overview of robust sub- space recovery," Proceedings of the IEEE, vol. 106, no. 8 , pp. 1380-1410, Aug 2018.

[14] H. Zou and L. Xue, "A selective overview of sparse principal component analysis," Proceedings of the IEEE, vol. 106, no. 8, pp. 1311-1320, 2018.

[15] J. He, L. Balzano, and A. Szlam, "Incremental gradient on the Grassmannian for online foreground and background separation in subsampled video," in IEEE Conf. Comput. Vis.Pattern Recogn. IEEE, 2012, pp. 1568-1575.

[16] J. Xu, V. K. Ithapu, L. Mukherjee, J. M. Rehg, and V. Singh, "GOSUS: Grassmannian online subspace updates with structured-sparsity," in IEEE Int. Conf. Comput. Vis., Dec 2013, pp. 3376-3383.

[17] F. Seidel, C. Hage, and M. Kleinsteuber, "pROST: a smoothed $\ell_{p}$-norm robust online subspace tracking method for background subtraction in video," Mach. Vis. Appl., vol. 25, no. 5, pp. 1227-1240, 2014.

[18] C. Hage and M. Kleinsteuber, "Robust PCA and subspace tracking from incomplete observations using $\ell_{0}$ surrogates," Computational Statistics, vol. 29, no. 3-4, pp. 467-487, 2014.

[19] J. Shen, H. Xu, and P. Li, “Online optimization for maxnorm regularization," in Advances in Neural Information Processing Systems, 2014, pp. 1718-1726.

[20] H. Mansour and X. Jiang, "A robust online subspace estimation and tracking algorithm," in IEEE Int. Conf. Acoust. Speech Signal Processing. IEEE, 2015, pp. 40654069.

[21] S. Chouvardas, Y. Kopsinis, and S. Theodoridis, "An adaptive projected subgradient based algorithm for robust subspace tracking," in IEEE Int. Conf. Acoust. Speech Signal Processing, 2014, pp. 5497-5501.

[22] $\_$, "Robust subspace tracking with missing entries: The set-theoretic approach," IEEE Transactions on Signal Processing, vol. 63, no. 19, pp. 5060-5070, 2015.

[23] J. Zhan, B. Lois, H. Guo, and N. Vaswani, “Online (and offline) robust PCA: Novel algorithms and performance guarantees," in Artif. Intell. Stat., 2016, pp. 1488-1496.

[24] B. Hong, L. Wei, Y. Hu, D. Cai, and X. He, "Online robust principal component analysis via truncated nuclear norm regularization," Neurocomputing, vol. 175, pp. 216222, 2016

[25] K. G. Quach, C. N. Duong, K. Luu, and T. D. Bui, “Nonconvex online robust PCA: Enhance sparsity via $\ell_{p}$-norm minimization," Computer Vision and Image Understanding, vol. 158, pp. 126-140, 2017.

[26] P. P. Markopoulos, M. Dhanaraj, and A. Savakis, “Adaptive 11-norm principal-component analysis with online outlier rejection," IEEE Journal on Selected Topics Signal Processing, vol. 12, no. 6, pp. 1131-1143, 2018.

[27] N. Linh-Trung, V. D. Nguyen, M. Thameri, T. MinhChinh, and K. Abed-Meraim, "Low-complexity adaptive algorithms for robust subspace tracking," IEEE Journal on Selected Topics Signal Processing, vol. 12, no. 6, pp. 11971212, 2018.

[28] P. Narayanamurthy and N. Vaswani, "Provable dynamic robust PCA or robust subspace tracking," IEEE Transactions on Information Theory, vol. 65, no. 3, pp. 1547-1577, March 2019.

[29] P. Narayanamurthy, V. Daneshpajooh, and N. Vaswani, "Provable subspace tracking from missing data and matrix completion," IEEE Transactions on Signal Processing, pp. 4245-4260, 2019.

[30] Y. Liu, K. Tountas, D. A. Pados, S. N. Batalama, and M. J. Medley, "L1-subspace tracking for streaming data," Pattern Recognition, vol. 97, p. 106992, 2020.

[31] X. Jia, X. Feng, W. Wang, H. Huang, and C. Xu, “Online schatten quasi-norm minimization for robust principal component analysis," Inf. Sci., vol. 476, pp. 83-94, 2019.

[32] P. Narayanamurthy and N. Vaswani, "Fast robust subspace tracking via PCA in sparse data-dependent noise," IEEE Journal on Selected Areas in Information Theory, vol. 1, no. 3, pp. 723-744, 2020. 
[33] R. Chakraborty, S. Hauberg, and B. C. Vemuri, "Intrinsic Grassmann averages for online linear and robust subspace learning," in IEEE Conf. Comput. Vis. Pattern Recogn., 2017, pp. 6196-6204.

[34] R. Chakraborty, L. Yang, S. Hauberg, and B. Vemuri, "Intrinsic Grassmann averages for online linear, robust and nonlinear subspace learning," IEEE Transactions on Pattern Analysis and Machine Intelligence, pp. 1-1, 2020.

[35] L. T. Thanh, N. V. Dung, N. L. Trung, and K. Abed Meraim, "Robust subspace tracking with missing data and outliers via ADMM," in European Signal Processing Conf., 2019, pp. 1-5.

[36] _ - "Robust subspace tracking with missing data and outliers: Novel algorithm with convergence guarantee," IEEE Transactions on Signal Processing, vol. 69, pp. 20702085,2021

[37] S. Hauberg, A. Feragen, R. Enficiaud, and M. J. Black, "Scalable robust principal component analysis using Grassmann averages," IEEE Transactions on Pattern Analysis and Machine Intelligence, vol. 38, no. 11, pp. 22982311, 2016.

[38] L. Balzano, R. Nowak, and B. Recht, “Online identification and tracking of subspaces from highly incomplete information," in Annual Allerton Conf. Commun. Control Comput. IEEE, 2010, pp. 704-711.

[39] Y. Chi, Y. C. Eldar, and R. Calderbank, "Petrels: Parallel subspace estimation and tracking by recursive least squares from partial observations," IEEE Transactions on Signal Processing, vol. 61, no. 23, pp. 5947-5959, Dec 2013.

[40] B. Yang, "Projection approximation subspace tracking," IEEE Transactions on Signal Processing, vol. 43, no. 1, pp. 95-107, 1995.

[41] Shing-Chow Chan, Yu Wen, and Ka-Leung Ho, "A robust past algorithm for subspace tracking in impulsive noise," IEEE Transactions on Signal Processing, vol. 54, no. 1, pp. 105-116, 2006.

[42] J. Zhang and T.-s. Qiu, "A robust correntropy based subspace tracking algorithm in impulsive noise environments," Digital Signal Processing, vol. 62, pp. 168-175, 2017.

[43] S. Luan, T. Qiu, L. Yu, J. Zhang, A. Song, and Y. Zhu, "BNC-based projection approximation subspace tracking under impulsive noise," IET Radar Sonar Navig., vol. 11, no. 7, pp. 1055-1061, 2017.

[44] B. Liao, Z. Zhang, and S.-C. Chan, "A new robust Kalman filter-based subspace tracking algorithm in an impulsive noise environment," IEEE Trans. Circuits Syst. II Express Briefs, vol. 57, no. 9, pp. 740-744, 2010.

[45] K. L. Blackard, T. S. Rappaport, and C. W. Bostian, "Measurements and models of radio frequency impulsive noise for indoor wireless communications," IEEE J. Sel. Areas Commun., vol. 11, no. 7, pp. 991-1001, 1993.

[46] W. J. Ebel and W. H. Tranter, "The performance of Reed-solomon codes on a bursty-noise channel," IEEE Transactions on Communications, vol. 43 , no. 2/3/4, pp. 298-306, 1995.

[47] Kung Yao, "A representation theorem and its applications to spherically-invariant random processes," IEEE Transactions on Information Theory, vol. 19, no. 5, pp. 600608, 1973

[48] E. Ollila, D. E. Tyler, V. Koivunen, and H. V. Poor, "Complex elliptically symmetric distributions: Survey, new results and applications," IEEE Transactions on Signal Processing, vol. 60, no. 11, pp. 5597-5625, 2012.

[49] C. L. Nikias and M. Shao, Signal processing with alphastable distributions and applications. Wiley-Interscience, 1995.

[50] P. G. Georgiou, P. Tsakalides, and C. Kyriakakis, "Alphastable modeling of noise and robust time-delay estimation in the presence of impulsive noise," IEEE Transactions on Multimedia, vol. 1, no. 3, pp. 291-301, 1999.

[51] J. Zhang, Q. Tian-shuang, and L. Sen, “A robust PAST algorithm based on maximum correntropy criterion for impulsive noise environments," Acta Electonica Sinica, vol. 43, no. 3, p. 483, 2015.

[52] J. Zhang and T. Qiu, "A novel tracking method for fast varying subspaces in impulsive noise environments," in 2016 Int. Conf. Signal Processing Commun. Syst., 2016, pp. $1-7$.

[53] T. Gustafsson, "Instrumental variable subspace tracking using projection approximation," IEEE Transactions on Signal Processing, vol. 46, no. 3, pp. 669-681, March 1998.

[54] G. Mercère, L. Bako, and S. Lecœuche, "Propagatorbased methods for recursive subspace model identification," Signal Processing, vol. 88, no. 3, pp. 468-491, 2008.

[55] S. Chan, H. Tan, and J. Lin, "A new variable forgetting factor and variable regularized square root extended instrumental variable PAST algorithm with applications," IEEE Trans. Aerosp. Electron. Syst., vol. 56, no. 3, pp. 18861902, 2020.

[56] Minhua Chen and Zuoying Wang, "Subspace tracking in colored noise based on oblique projection," in IEEE Int. Conf. Acoust. Speech Signal Processing, vol. 3, 2006, pp. 556-559.

[57] F. Yger, M. Berar, G. Gasso, and A. Rakotomamonjy, "Oblique principal subspace tracking on manifold," in IEEE Int. Conf. Acoust. Speech Signal Processing, 2012, pp. $2429-2432$.

[58] S. Chan, Z. Zhang, and Y. Zhou, "A new adaptive kalman filter-based subspace tracking algorithm and its application to DOA estimation," in IEEE Int. Symp. Circuits Systt., 2006, pp. 4 pp.-132.

[59] Chuang Wang and Y. M. Lu, "Online learning for sparse PCA in high dimensions: Exact dynamics and phase transitions," in IEEE Inf. Theory Works., 2016, pp. 186190.

[60] W. Yang and H. Xu, "Streaming sparse principal component analysis," in Int. Conf. Mach. Learn., 2015, pp. 494503.

[61] X. Yang, Y. Sun, T. Zeng, T. Long, and T. K. Sarkar, “Fast STAP method based on PAST with sparse constraint for airborne phased array radar," IEEE Transactions on Signal Processing, vol. 64, no. 17, pp. 4550-4561, 2016.

[62] P. V. Giampouras, A. A. Rontogiannis, K. E. Themelis, and K. D. Koutroumbas, "Online sparse and low-rank subspace learning from incomplete data: A Bayesian view," Signal Processing, vol. 137, pp. 199-212, 2017.

[63] N. Lassami, K. Abed-Meraim, and A. Aïssa-El-Bey, "Low cost subspace tracking algorithms for sparse systems," in European Signal Processing Conf. IEEE, 2017, pp. 14001404.

[64] N. Lassami, A. Aïssa-El-Bey, and K. Abed-Meraim, “Low cost sparse subspace tracking algorithms," Signal Processing, vol. 173, p. 107522, 2020.

[65] R. Badeau, G. Richard, and B. David, "Fast and stable YAST algorithm for principal and minor subspace tracking," IEEE Transactions on Signal Processing, vol. 56, no. 8, pp. 3437-3446, Aug 2008.

[66] Z. Zhang, Y. Xu, J. Yang, X. Li, and D. Zhang, "A survey of sparse representation: Algorithms and applications," IEEE Access, vol. 3, pp. 490-530, 2015.

[67] T. T. Cai, Z. Ma, Y. Wu et al., "Sparse PCA: Optimal rates and adaptive estimation," The Annals of Statistics, vol. 41, no. 6, pp. 3074-3110, 2013.

[68] D. Papailiopoulos, A. Dimakis, and S. Korokythakis, "Sparse PCA through low-rank approximations," in Int. Conf. Mach. Learn., 2013, pp. 747-755.

[69] V. Q. Vu, J. Cho, J. Lei, and K. Rohe, "Fantope projection and selection: A near-optimal convex relaxation of sparse PCA," in Advances in Neural Information Processing Systems, 2013, pp. 2670-2678.

[70] N. Lassami, A. Aïssa-El-Bey, and K. Abed-Meraim, “Fast sparse subspace tracking algorithm based on shear and givens rotations," in Asilomar Conf. Signals Syst. Comput., 2019, pp. 1667-1671. 


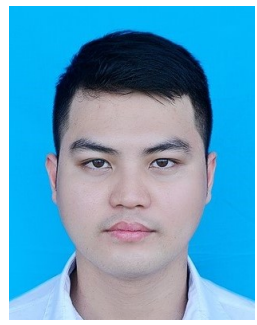

Le Trung Thanh received B.Sc. and M.Sc. degrees in Electronics and Telecommunications from VNU University of Engineering and Technology, Vietnam National University, Hanoi (VNU) in 2016 and 2018 respectively. He is now pursuing his Ph.D. study at the University of Orleans, France. His research interests include signal processing, subspace tracking, tensor analysis, and system identification.

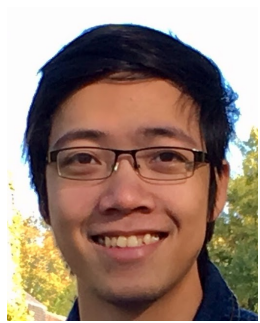

Nguyen Viet Dung received the B.Sc. in Electronics and Telecommunication Engineering from VNU University of Engineering and Technology, Vietnam National University, Hanoi (VNU) in 2009, M.Sc. in Networks and Telecommunications from École Normale Supérieure (ENS) de Cachan, Université Paris XI (now, University of Paris-Saclay, France), in 2012, and Ph.D. in Signal Processing from the University of Orleans (France) in 2016. He did a postdoc at CentraleSupélec, University of Paris-Saclay from 2017 to 2018. From 2019 to now, he is a research engineer at Lab-STICC, UMR 6285 CNRS ENSTA Bretagne, Brest, France. His research interests include channel modelling in array signal processing, adaptive matrix and tensor analysis, blind source separation, and statistical performance analysis.

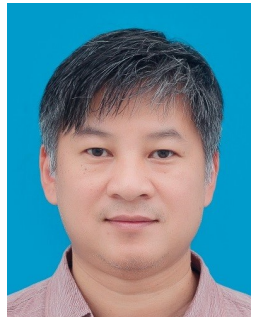

Nguyen Linh Trung obtained his B.Eng. and Ph.D. degrees, both in Electrical Engineering, from Queensland University of Technology, Brisbane, Australia, in 1998 and 2005. Since 2006, he has been on the faculty of VNU University of Engineering and Technology, Vietnam National University, Hanoi (VNU), where he is currently an associate professor of electronic engineering in the Faculty of Electronics and Telecommunications and director of the Advanced Institute of Engineering and Technology. He is interested in signal processing methods, including time-frequency signal analysis, blind processing, adaptive filtering, compressive sampling, tensor-based signal analysis, graph signal processing, and apply them to wireless communication, networking, and biomedical engineering.

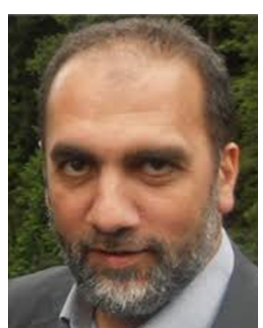

Karim Abed-Meraim was born in 1967. He received the State Engineering Degree from Ecole Polytechnique, Palaiseau, France, in 1990, the State Engineering Degree from Ecole Nationale Supérieure des Télécommunications (ENST), Paris, France, in 1992, the M.Sc. degree from Paris XI University, Orsay, France, in 1992 and the Ph.D. degree from the ENST in 1995 (in the field of Signal Processing and communications). From 1995 to 1998 he took a position as a research staff at the Electrical Engineering Department of the University of Melbourne where he worked on several research project related to "Blind System Identification for Wireless Communications", "Blind Source Separation", and "Array Processing for Communications", respectively. From 1998 to 2012 he has been Assistant then Associate Professor at the Signal and Image Processing Department of Telecom-ParisTech. His research interests are in signal processing for communications, adaptive filtering and tracking, array processing and statistical performance analysis. In September 2012 he joined the University of Orleans (PRISME Lab.) as a full Professor. He is the author of over 450 scientific publications including book chapters, international journal and conference papers and patents. Dr. Abed-Meraim is an IEEE Fellow, an IEEE SAM-TC member and a past associate editor for the IEEE Transactions on Signal Processing. 